\title{
Surface meteorological instrumentation for BOBMEX
}

\author{
G S Bhat and S AmeEnulla \\ Centre for Atmospheric and Ocean Sciences, Indian Institute of Science, Bangalore 560 012, India
}

\begin{abstract}
Although India has a long experience in ship-borne experiments and oceanographic instrumentation, the atmospheric component has not received much attention in the past. In this paper, the basis of the atmospheric instrumentation system assembled for use on board ORV Sagar Kanya for the BOBMEXPilot experiment is described along with some representative results. Wherever possible, Woods Hole's IMET recommendations for meteorological sensors for applications in the marine environment have been followed to keep our measurements in par with international standards. The sensors were tested during the BOBMEX-Pilot experiment and all sensors worked well. Velocity, humidity and temperature data have been successfully collected using fast sensors. It is shown that the component due to the ship's pitching motion can be removed from the measured vertical velocity by making use of an accelerometer. This makes it possible to calculate the surface fluxes by direct methods.
\end{abstract}

\section{Introduction}

One major emphasis of the Indian Climate Research Programme (ICRP) is on the physical processes taking place during the summer monsoon in the atmosphere and the ocean over the warm seas that surround the Indian sub-continent. For this purpose, several ocean-based field experiments of four to six weeks duration have been planned, the Bay of Bengal and Monsoon Experiment (BOBMEX) and the Arabian Sea Monsoon Experiment (ARMEX) being two examples (ICRP Implementation Plan 1998). The main components of these experiments will be carried out on board research ships. The exchange of mass, momentum and energy between the ocean and the atmosphere takes place at the sea surface and accurate measurement of near surface meteorological parameters is necessary to calculate the interface fluxes. However, this is a difficult task because of high humidity levels, sea spray and the movement of the platform from where measurements are made. In addition, the sensors and instruments have to work in the harsh marine environment without requiring service support. India has a long experience in shipborne experiments and oceanographic instrumentation, however, the atmospheric component has not received much attention in the past. Therefore, one important task was to set up an instrumentation system for the measurement of surface meteorological parameters on board research ships. In this paper, the atmospheric instrumentation system assembled for use on board ORV Sagar Kanya for the BOBMEXPilot experiment, the first field campaign under ICRP, is described along with some representative results.

\section{Sensor selection}

Important surface meteorological parameters include pressure, wind speed and wind direction, air temperature, humidity, radiation and rainfall. The accuracy requirements on these parameters is dictated by the application such as the acceptable uncertainty on surface fluxes for use in numerical models. The precise accuracy requirements on the above parameters have not been worked out in the Indian context. For the International programs such as WOCE (World Ocean Circulation Experiment) and TOGA (Tropical Ocean Global Atmosphere), the absolute accuracy of various parameters have been arrived at in order to achieve heat flux accuracy of $10 \mathrm{~W} / \mathrm{m}^{2}$ for each component of energy balance (table 1) (Large 1985; Hosom et al

Keywords. Meteorological instrument; air-sea flux. 
Table 1. Accuracies desired in surface meteorological data collected for the World Circulation Experiment (Large 1985; Hosom et al 1995). The numbers include all sources of error.

\begin{tabular}{ll}
\hline Variable & Desired accuracy \\
\hline Wind speed & Larger of $2 \%$ or $0.2 \mathrm{~m} / \mathrm{s}$ \\
Wind direction & $2.8^{\circ}$ \\
Air temperature & $0.25^{\circ} \mathrm{C}$ \\
Incoming short wave radiation & Better than $10 \mathrm{~W} / \mathrm{m}^{2}$ \\
Incoming long wave radiation & Better than $10 \mathrm{~W} / \mathrm{m}^{2}$ \\
Relative humidity & $1.7 \%$ \\
Specific humidity & $0.25 \mathrm{~g} / \mathrm{kg}$ \\
Precipitation & $1 \mathrm{~cm}$ per month \\
Barometric pressure & $1 \mathrm{mb}$ \\
\hline
\end{tabular}

1995). The numbers given in table 1 are absolute accuracies, i.e., includes that of sensor, interface electronics, signal transmission and data acquisition. Such accuracies are required for getting the uncertainties in the predictions of climate models within acceptable limits, and by no way mean that all are achievable in practice.

The selection of each component of the measurement system consisting of the sensors, signal cables, interface electronics and data acquisition procedure is very crucial in achieving high levels of accuracy. A survey of the available sensors revealed that, in principle, several makes could meet the above accuracy specifications, and the main question would be their performance and reliability under marine conditions. Considerable time and a lot of resources are needed to make field trials and to decide on the best among the available makes. Given the time frame of our programmes, this is not possible and therefore we considered the experience of others involved in the earlier land and ocean based experiments. A group at Woods Hole Oceanographic Institution (WHOI), USA has developed IMET (Improved Meteorology) system for use on-board in ship and buoy (Hosom et al 1995). A series of performance and reliability tests under real marine conditions were carried out by them on meteorological sensors made by well known companies. These tests lasted for a period of about two years. Based on the outcome, IMET system has been configured (Hosom et al 1995). In the IMET system, the best performing wind, temperature, humidity, radiation, pressure and precipitation sensors have been integrated into one complete system along with the data acquisition system. The makes recommended by WHOI and used in the IMET system are shown in table 2 . It is seen from table 2 that R M Young products are recommended for wind speed, wind direction and precipitation, Eppley Lab for the radiation, and Rotronic for humidity. The IMET system has been extensively used during the TOGA observations. The IMET system is sold by WHOI, however, the entire system is very expensive. The same sensors are directly available from manufacturers at a much cheaper price, and using the sensors recommended by WHOI wherever possible and integrating them into a complete system turned out to be considerably less expensive and was followed while developing the atmospheric instrumentation for the BOBMEX programme.

We would like to stress here that the IMET system developed by WHOI includes only slow sensors, i.e., sensors whose response times are few seconds or more. For meeting certain objectives of BOBMEX, namely the direct estimation of surface heat and momentum fluxes, wind, temperature and humidity sensors need to have a frequency response of at least 5 to $10 \mathrm{~Hz}$ (fast sensors). Since a study comparable to IMET has not been carried out for the fast sensors, the decision was based on the experience in our recent national experiments, namely MONTBLEX-90 and land surface processes experiment at Anand (personal communication, Sri. K G Vernekar, IITM) along with the overall sensor design. The basic surface met sensors selected for BOBMEX are shown in table 3. (Surface pressure was manually recorded from the pressure gauge on board the ship). From tables 2 and 3 we see that by and large, the slow sensors selected for BOBMEX are the same as those used in the IMET system except the humidity sensor. In the IMET system, Rotronics humidity sensor is used along with $\mathrm{R} M$ Young radiation shield whereas in the BOBMEX set up, R M Young humidity sensor with R M Young radiation shield is used (Rotronics sensor might have required certain modifications in the radiation shield, and on compatibility considerations $\mathrm{R}$ M Young humidity sensor was selected). We have used R M Young humidity sensor during INDOEX cruises and found it to be accurate and it worked well in the marine environment.

The measurement of humidity, especially from a fast response sensor, has been a major problem in the previous field experiments in India. In previous experiments, Lyman alpha sensor had been used and was found to have problems when it rained. In the present set up, we have selected the infrared (IR) hygrometer as the fast response humidity sensor. This instrument operates on the principle of electromagnetic radiation absorption characteristics of water vapor. It consists of a light source and a detector enclosed in two sealed housings, that are separated by

Table 2. Woods Hole Oceanographic Institution recommendations for ship and buoy MET sensors.

\begin{tabular}{ll}
\hline Variable & Make \\
\hline Wind speed & R M Young \\
Wind direction & R M Young \\
Air temperature & PRT with R M Young shield \\
Incoming short wave radiation & Eppley \\
Incoming long wave radiation & Eppley \\
Relative humidity & Rotronic \\
Specific humidity & - \\
Precipitation & R M Young \\
\hline
\end{tabular}


Table 3. Sensors used in the system developed for ICRP ship based field experiments.

\begin{tabular}{lll}
\hline Parameter & \multicolumn{1}{c}{ Accuracy } & \multicolumn{1}{c}{ Make } \\
\hline Slow Sensors & & \\
Air temperature & $0.2^{\circ} \mathrm{C}$ & PRT, R M Young radiation shield \\
Humidity & $3 \% \mathrm{RH}$ & R. M. Young \\
Incoming short wave & $\sim 10 \mathrm{~W} / \mathrm{m}^{2}$ & Eppley \\
Incoming long wave & $\sim 10 \mathrm{~W} / \mathrm{m}^{2}$ & Eppley \\
Precipitation & $1 \mathrm{~mm}$ & R M Young \\
Fast sensors $(\mathbf{1 0 ~ H z}$ or better) & & \\
3-D wind velocity & $0.1 \mathrm{~m} / \mathrm{s}($ Sonic $)$ & METEK Germany \\
3-D wind velocity & $1 \%(\mathrm{Gill}$ propeller) & R M Young \\
Temperature & $0.01^{\circ} \mathrm{C}($ Sonic $)$ & METEK Germany \\
Humidity & $0.5 \mathrm{~g} / \mathrm{m}^{3}$ & Applied Technologies, USA \\
Acceleration & $0.05 \mathrm{~m} / \mathrm{s}^{2}$ & Crossbow, USA \\
\hline
\end{tabular}

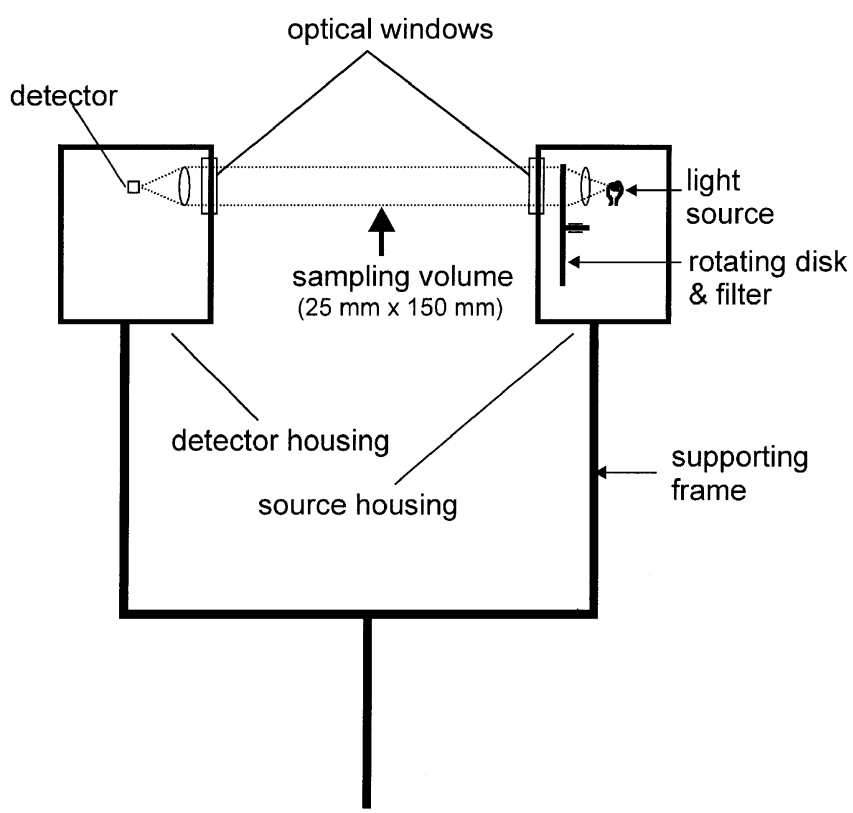

Figure 1. A schematic of the IR hygrometer sensor assembly.

an open space that forms the sampling volume (figure 1). An optical window, in each housing enables the source radiation to pass through the air gap and fall on the detector at the other end. The volume of the air sampled is $25 \mathrm{~mm}$ (1") in diameter and $0.15 \mathrm{~m}$ long. A rotating (3000 rpm) disk, placed in front of the light source and having two optical filters fixed on it at certain angular positions, alternately makes the water vapor absorbing and water vapor transparent radiation from the same source to pass through the sampling volume. The amount of water vapor present in the air column along the beam path is calculated from the ratio of the intensities of radiation measured by the detector at these two frequencies. The main advantage of this arrangement is that all components are completely sealed (sensor not spoilt by rain or sea spray), and any deposition on the mirror surface or change in the light source intensity will be felt equally at both the frequencies and is effectively removed when their ratio is taken. This design is rugged and suitable for marine applications.

\section{Signal transmission and interface electronics}

The data acquisition system was located in the Met laboratory at a distance of about 80 meters from sensors. Shielded cables have been used to transmit the signals from the sensors to reduce the interference from other sources. The multi-core, shielded nylon cables are very good for such purposes and have been used during BOBMEX-Pilot. The output of some of the sensors were RS232 (IR hygrometer) or RS422 (sonic anemometer) digital signals, whereas, others analogue. The RS422 signal was converted to RS232 signal in the Met laboratory. Digital signals were directly fed to the PC through a multi-serial port addon PC card. Analogue signals were digitized before being fed to the data acquisition system.

When the cable lengths are long (e.g., 90-100 meters as in the present case), precaution is required in measuring the sensor output voltage because of the loop current. Sensors draw a small current and the voltage drop between the two ends of the connecting cable can become important if the cable lengths are long. In such cases, the calibration constants obtained in the laboratory using short cables (typically few meters) will not be valid. Also, for each parameter, two channels (representing high and low ends of the sensor output) of the A/D converter are required. Thus, if the number of signals are large (as in the present case), then the demand on the capacity of the data acquisition board becomes severe. A solution to both these problems is an electronics circuitary (interface electronics) based on operational amplifier, whose schematic is shown in figure 2. Outputs (high and low) from each sensor were transmitted to the laboratory using cables independent of that used for driving the sensor, added up and output as one signal with respect to a common ground in the laboratory. 


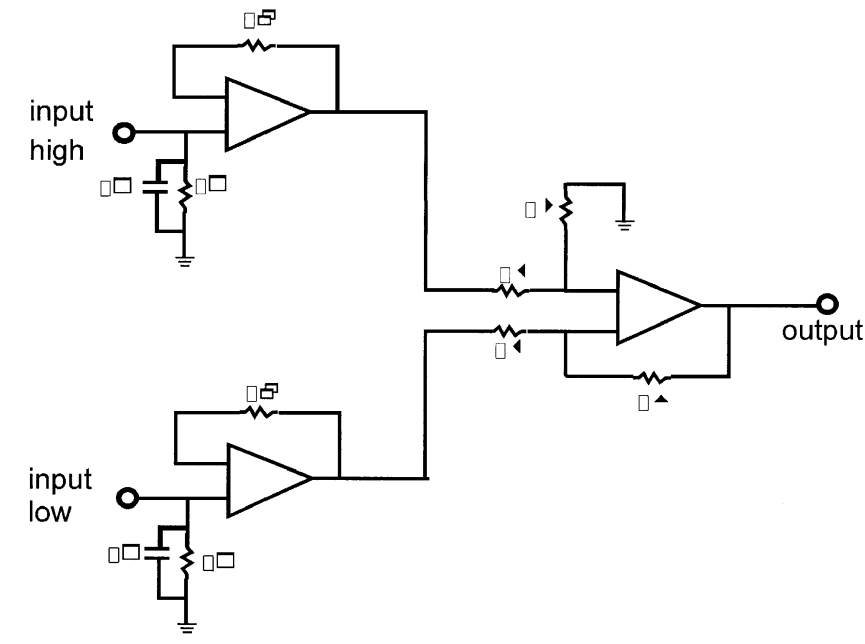

Figure 2. Schematic of the interface electronics for measuring the sensor outputs relative to a common ground.

Wherever required, additional signal conditioning facility was incorporated in the circuit to suit individual sensor characteristics. The outputs from the interface electronics were fed to the data acquisition system, a 12 bit system having 16 single ended analogue input channels (DAQbook). A PC working on Windows 95 was used for acquiring and storing the data. Data acquisition and archival were controlled by software. This program handled the data from the DAQbook and RS232 digital inputs coming from sonic anemometer and IR Hygrometer, as well as the display of all the parameters on the screen for visual monitoring in real time.

\section{Sensor testing}

Most of the sensors were new and calibrated at the factory. Since the marine atmosphere is very corrosive and the sensors can get degraded, all sensors have been tested after the field experiment. The radiation instruments have been tested at the Central Radiation Laboratory, IMD Pune and were found to maintain the factory calibrated sensitivity. Wind, temperature and humidity sensors have been tested in the laboratories at Indian Institute of Science, Bangalore. For example, figure 3 shows the performance of Gill and Sonic anemometers tested in the two feet wind tunnel after the Pilot experiment. One component of the sensor was aligned along the mean wind direction, wind speed increased from about $3 \mathrm{~m} / \mathrm{s}$ to $15 \mathrm{~m} / \mathrm{s}$ and then decreased back (steady wind speeds below $4 \mathrm{~m} / \mathrm{s}$ are difficult to maintain and less accurate). The results shown are the averages of two sets for each component. There is good agreement between the wind tunnel, sonic and Gill anemometer wind speeds. The overall error includes that of alignment and wind speed setting, in addition to sensor accuracy. In our opinion, much of the scatter seen in figure 3 is due

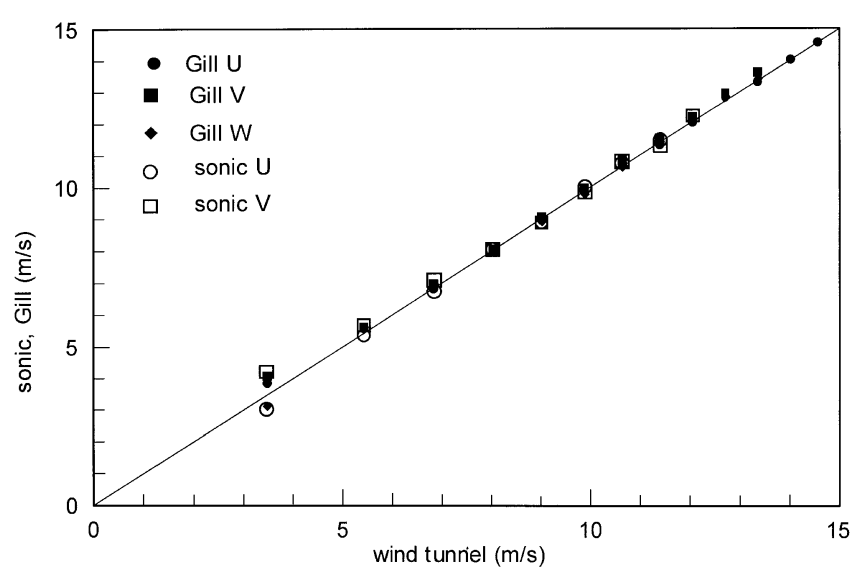

Figure 3. Comparison of Gill and Sonic anemometer measured wind speeds with the wind tunnel wind speed.

to alignment and wind speed setting problems. The testing confirmed that the performance of Sonic and Gill anemometers have not been degraded after they were used in the Pilot experiment.

Temperature and humidity sensors have been tested in a closed, insulated chamber where the relative humidity is maintained constant using saturated salt solutions and the temperature was controlled by circulating water from a constant temperature bath. These sensors have been tested using connecting cables used in the field experiment. The humidity sensor's response was in good agreement with the original specifications, however a change in the sensitivity of the temperature sensor was observed (from $20^{\circ} \mathrm{C} / \mathrm{V}$ to $21^{\circ} \mathrm{C} / \mathrm{V}$ ) and the new values have been used in the preparation of the Pilot data set.

The sonic anemometer measures the virtual temperature of the air, i.e., the temperature corrected for the presence of water vapor. Our calibration confirmed this, however, a constant offset between the reference virtual temperature and sonic temperature was noted. The reason for this is that by an option in the sonic anemometer set up menu, the mean temperature can be set to the dry bulb temperature. This way, the mean temperature corresponds to the air temperature (approximately) and the fluctuating component gives the turbulent component of virtual temperature.

\section{Results}

The sensors were tried in the field during the BOBMEX-Pilot experiment on board ORV Sagar Kanya from 25th October to 12th November 1998. The sensors were mounted on a micrometeorological tower fixed at the end of a seven meter long horizontal boom at the forecastle deck of the ship (figure 4). The sensors on the tower were switched on from October 25th to November 8th, 1998. The data archival was controlled by the DASYLab software. Basically, the 


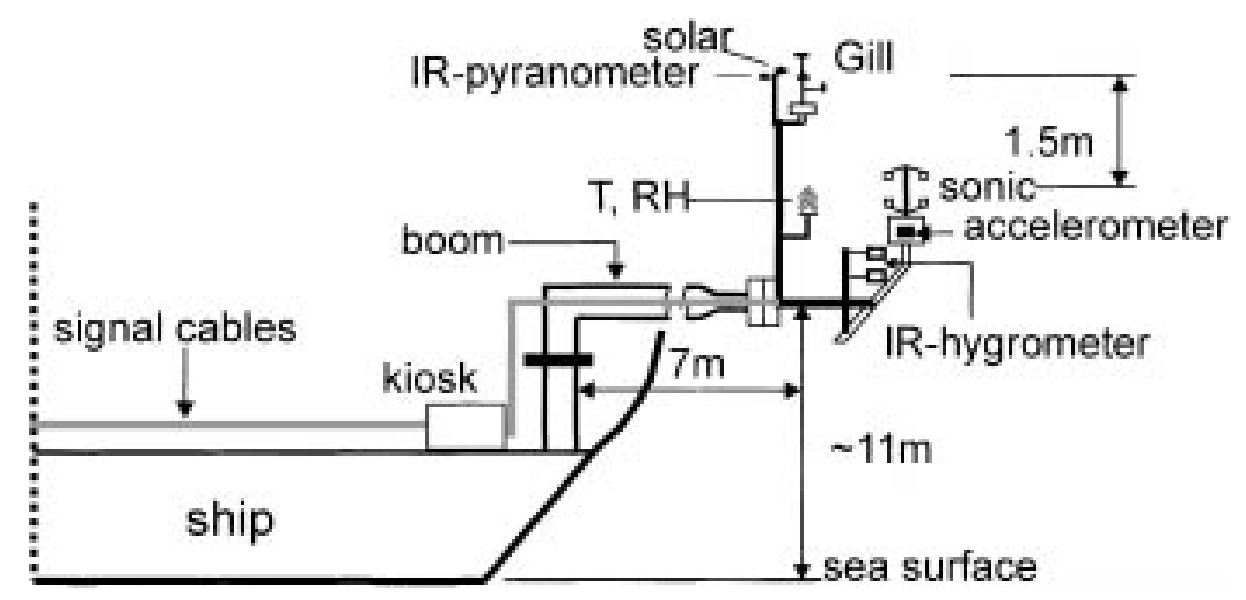

Figure 4. Arrangement of surface meteorological sensors on board ORV Sagar Kanya.

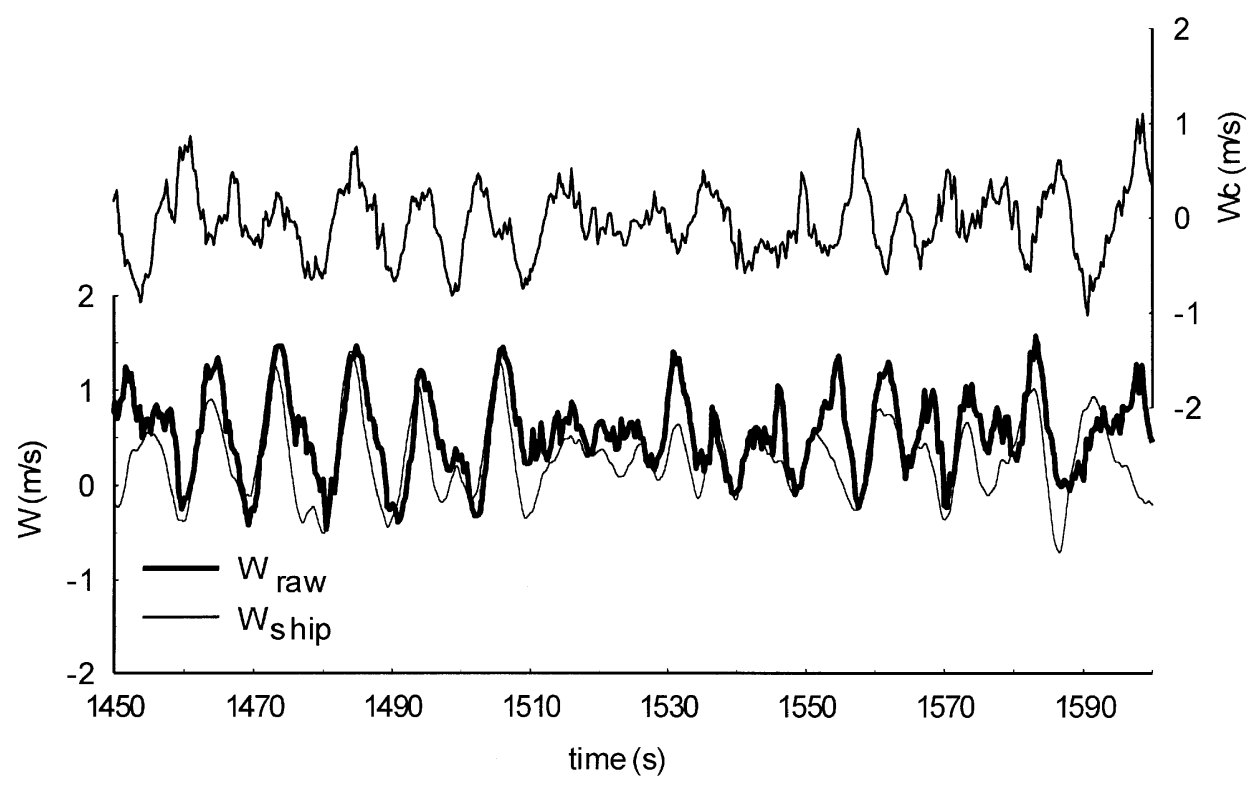

Figure 5. A short time series of the vertical velocity. $W_{\text {raw }}$ is the vertical velocity measured by the sonic anemometer, $W_{\text {ship }}$ is vertical velocity caused by the ship's pitching motion calculated from the accelerometer data and $W_{\mathrm{c}}$ is the corrected vertical velocity.

so called fast data $(10 \mathrm{~Hz}$ sampling rate) was acquired for 65 minutes once in every three hours $(00,03,06$, 09, 12, 15, 18 and 21 hours IST) and stored in separate files. The data from the fast sensors were averaged for 10 seconds and these values were stored continuously. The data from the slow sensors $(1 \mathrm{~Hz}$ sampling frequency) were averaged for 10 seconds and also archived continuously. In order to limit the size of individual files, one set of files was closed and a new set of files was opened every three hours automatically. All the sensors, interface electronics and the data acquisition system worked continuously without any problem for the entire duration of the Pilot experiment.

Results from the slow sensor data are presented in a companion paper in this volume (Bhat et al 2000) wherein we present representative data from fast sensors. One main application of the fast data is in the estimation of the fluxes by the eddy correlation method. This involves computing $v^{\prime} w^{\prime}, q^{\prime} w^{\prime}, T^{\prime} w^{\prime}$, etc., where $v$ and $w$ are respectively horizontal and vertical components of wind velocity, and $q$ and $T$ are mixing ratio of water vapor and air temperature respectively; the primes denote the deviations from respective 15 to 20 minutes average values. Accurate measurement of vertical component of velocity is very important in these calculations. However, at the sampling rate of $10 \mathrm{~Hz}$, measured $w$ is strongly influenced by the rolling and pitching motion of the ship. An accelerometer was mounted on the stem of the sonic anemometer to measure the movement of the sensor caused by the ship's motion. Figure 5 shows the vertical velocity measured by the sonic anemometer $\left(W_{\text {raw }}\right)$, calculated from the accelerometer output ( $\left.W_{\text {ship }}\right)$ and the difference between the two, namely the corrected vertical velocity $\left(W_{\mathrm{c}}\right)$. It is 


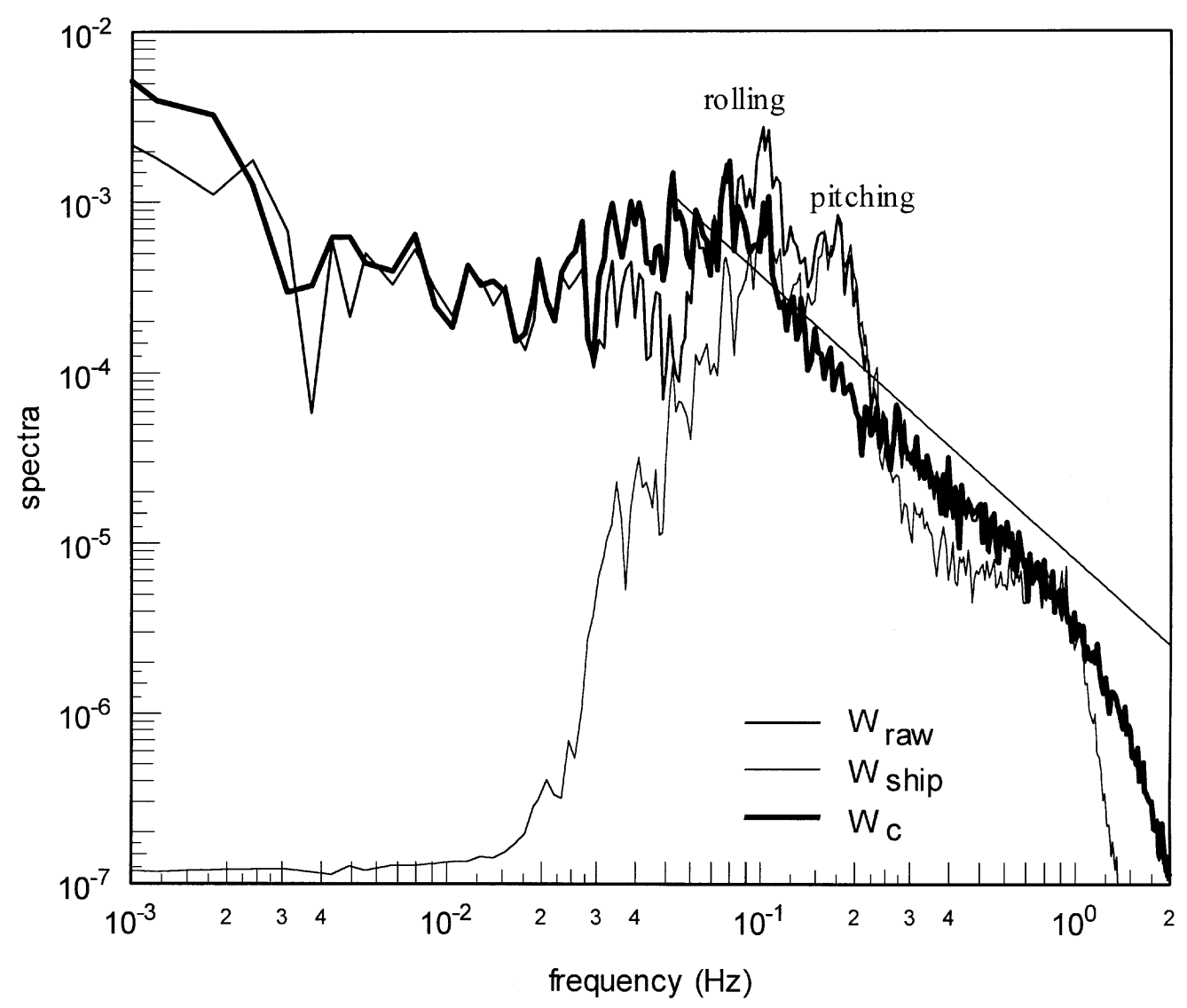

Figure 6. The spectra of vertical velocities. The thin straight line corresponds to $f^{-5 / 3}$ decay rate.

observed from figure 5 that large fluctuations in the vertical velocity measured by the sonic anemometer are caused by the ship motion itself and the actual vertical velocity of the air $\left(W_{\mathrm{c}}\right)$ has a different appearance. This is clearly seen in the spectra of three velocities shown in figure 6 . The spectrum of $W_{\text {raw }}$ has two peaks near $0.1 \mathrm{~Hz}$ corresponding to the pitching and rolling motions of the ship. In the spectrum of the corrected vertical velocity, the component due to the pitching motion of the ship has been very successfully removed. The peak at the rolling frequency has been reduced by a factor of about five, but not completely removed. This encourages the hope that it is possible to obtain wind vertical velocity on board the ships with reasonable confidence.

In a fully developed turbulent flow, the spectra of velocity and scalars follow the Kolmogorov's $k^{-5 / 3}$ decay law over a range of wave number $k$ at high wave numbers (inertial subrange) (Batchelor 1953). The wave number is proportional to the frequency $f$, and therefore the spectrum is proportional to $f^{-5 / 3}$ in the inertial subrange in figure 6 . It is observed from figure 6 that the range of frequencies where the $k^{-5 / 3}$ decay law prevails is enhanced after the correction to the ship's motion. The question is, how important is the correction for $w$ in the estimation of fluxes? Our calculations showed that the contamination due to
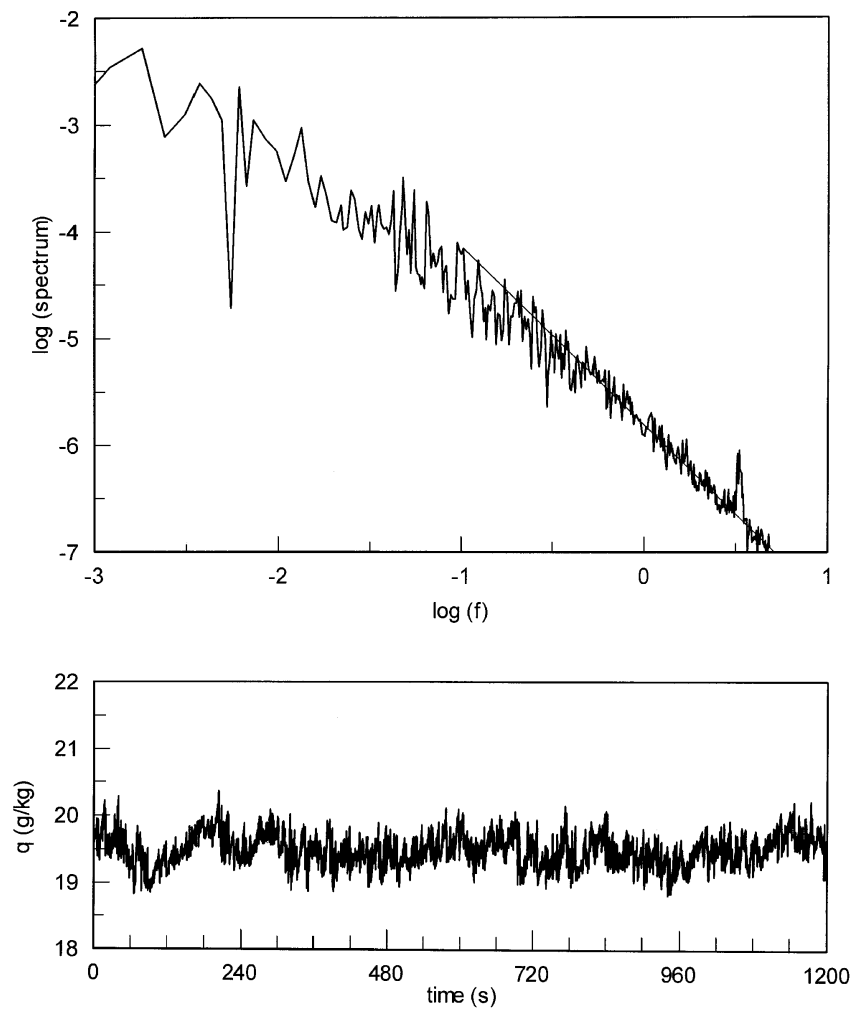

Figure 7. Time series (bottom) and spectrum (top) of mixing ratio $q$ measured by the IR Hygrometer. The thin straight line in the top frame has a slope of $(-5 / 3)$. 
the ship's pitching on the estimated fluxes is substantial when swell waves are strong and wind speeds less than $3 \mathrm{~m} / \mathrm{s}$. For example, when swell waves were about 2 meters high, the calculated sensible heat fluxes are about $35 \mathrm{~W} / \mathrm{m}^{2}$ and $10 \mathrm{~W} / \mathrm{m}^{2}$ respectively using uncorrected and corrected vertical velocities, the latter looks very reasonable over the oceans (these results will be reported in a separate paper).

Figure 7 shows the data from the IR hygrometer sampled at $10 \mathrm{~Hz}$. According to theory, the spectrum of the scalars should follow the $k^{-5 / 3}$ decay law at higher frequencies in a turbulent flow, and the data from the hygrometer are in good agreement with the theory suggesting that the frequency response of the hygrometer is pretty good.

\section{Conclusion}

The surface Met instrumentation for measurements on board ship for ICRP has been developed. Wherever possible, Woods Hole's IMET recommendations have been followed. The sensors were tested during the BOBMEX-Pilot experiment and all sensors worked well. Post field experiment testing has confirmed that there was no noticeable degradation in the performance of the sensors. Velocity, humidity and temperature data have been successfully obtained using fast sensors. It is shown that the component due to the ship's pitching motion can be removed from the measured vertical velocity by making use of an accelerometer. The rolling component is not completely removed and additional sensors like tilt sensors that measure the roll angle may help in determining the contamination due to rolling.

\section{Acknowledgements}

This work was supported by a grant from the Department of Science and Technology, New Delhi and we thank the agency for the support. We thank Antarctica Study Centre (ASC), Goa for making available the ship along with its facilities for the Pilot experiment. We thank Prof. A Prabhu, Sri K G Vernekar and Dr. K Sengupta for many useful discussions and suggestions. We thank Dr. Kartik Venkataraman for sparing the wind tunnel for testing the wind sensors.

\section{References}

Batchelor G K 1953 Theory of homogenous turbulence (Cambridge University Press)

Bhat G S, Ameenulla S, Venkataramana M and Sengupta K 2000 Proc. Indian Acad. Sci., (Earth Planet. Sci.) (this issue)

Hosom D S, Weller R A, Payne R E and Prada K E $1995 \mathrm{~J}$. Atmos. and Oceanic Tech. 12 527-540

Indian Climate Research Programme. Implementation Plan 1998 (New Delhi: Department of Science and Technology)

Large W G (ed.) World Ocean Circulation Experiment: U.S. WOCE 1985, Tech. Rep. No.1, WOCE Global Air-Sea Interaction Fields, $36 \mathrm{pp}$. 